\title{
Hétérogénéité et attentes différentielles: une approche de didactique comparée
}

\section{Francia Leutenegger et Serge Quilio}

Cette contribution propose de revisiter le concept d'hétérogénéité selon un éclairage didactique des phénomènes concernant les élèves déclarés "en difficulté». L'objectif est ici d'examiner comment, dans le collectif de classes ordinaires de 3P et 4P (HarmoS), interagissent enseignant et élèves à propos de tâches spécifiques (nombre et soustractions). Le concept de contrat didactique différentiel permet d'analyser le fonctionnement des interactions et la gestion enseignante. Sont particulièrement étudiées, l'articulation entre le traitement individuel des différences entre élèves et le traitement collectif par l'enseignant, et les effets sur l'apprentissage des élèves (quelques-uns) de cette gestion, plutôt individuelle versus dans le collectif de la classe.

A l'aide du concept de contrat didactique différentiel (Schubauer-Leoni, 1987) et des méthodes mises en ouvre dès le début des années 2000 par les travaux comparatistes en didactique (Mercier, Schubauer-Leoni \& Sensevy, 2002), nous décrirons l'action conjointe enseignant-élèves (au sens de l'ouvrage coordonné par Sensevy \& Mercier, 2007) dans le cadre de deux classes ordinaires, l'une de 3P, l'autre de $4 \mathrm{P}(\mathrm{HarmoS}$, élèves de 6 à 8 ans). La première est observée à propos d'une situation fondamentale concernant le nombre, la seconde à propos d'une séquence sur la soustraction. Dans chacune des classes, quelques élèves sont repérés par leur enseignante comme présentant des difficultés alors que le reste de la classe semble répondre à ses attentes.

Toutefois la présentation de chacune de ces études sera d'inégale importance. En effet, l'étude concernant la classe de $3 \mathrm{P}-\mathrm{H}$ a déjà fait l'objet d'une publication (Leutenegger, 2009) et nous ne ferons que résumer les résultats majeurs sur lesquels nous nous appuierons pour réaliser une étude comparative avec la classe de $4 \mathrm{P}$ présentant une autre gestion de l'hétérogénéité par l'enseignante. Ce sont les effets de ces différences de gestion sur l'apprentissage de quelques élèves que nous allons étudier. 


\section{Eléments théorico-méthodologiques pour L'analyse}

Avec un cadrage didactique, nous considérons, classiquement, le système triadique (enseignant-élèves-objet de savoir) comme unité d'observation. Ce sont les phénomènes inhérents au contrat didactique, aux attentes réciproques, pour la plupart implicites, entre enseignant et élèves à propos des savoirs qui retiendront notre attention. Dès les années 1980, Schubauer-Leoni (1987) a décrit la fonction du concept de contrat didactique à l'articulation entre les études sur les représentations sociales et celles du champ didactique. L'auteure allie une entrée par les savoirs à une entrée par les sujets didactiques (enseignants et élèves) et montre la nécessité du concept de contrat didactique différentiel qui permet d'articuler ce qui relève de l'individuel avec ce qui relève du collectif de la classe.

Dès lors, au plan de l'hétérogénéité, comment tenir compte à la fois de ce fonctionnement collectif et de la manière dont chaque élève s'approprie (ou non) les objets culturels de savoir proposés à l'école. La didactique comparée postule la nécessité de distinguer des positions différentielles dans ce collectif pour parvenir d'une part à montrer quels sont les apprentissages des élèves, mais aussi à comprendre le fonctionnement du système tout entier. C'est ce que nous tenterons de montrer à travers cette étude comparative, en nous focalisant sur l'enseignant et ses décisions et en cherchant ce que celles-ci produisent sur l'apprentissage des élèves, différentiellement.

Depuis les années 2000, une tentative de modélisation de l'action conjointe enseignant-élèves (Sensevy \& Mercier, 2007) selon plusieurs paramètres permet de décrire les formes de l'action des enseignants et des élèves, spécifiées par les conditions didactiques de rencontre avec les objets de savoir. Ce modèle comporte une double ambition comparatiste, qui a été décrite ailleurs (Ligozat $\&$ Leutenegger, 2008; Schubauer-Leoni, Leutenegger, Ligozat \& Fluckiger, 2007) et que nous rappellerons brièvement: l'enjeu concerne d'une part la possible mise en évidence de pratiques spécifiques etlou génériques (au sens de Mercier, Schubauer-Leoni \& Sensevy, 2002) entre différents enjeux de savoirs et, d'autre part, un travail sur les concepts théoriques tels que les notions d'activité, action, intention, pratiques, etc., développées notamment par les disciplines d'étude des dimensions du travail humain et portées par différents champs disciplinaires, tels que la psychologie, la sociologie, la philosophie analytique ou encore les sciences du langage. ${ }^{1}$

Dès la construction d'un premier modèle, Sensevy, Mercier et SchubauerLeoni (2000) ont retenu différentes dimensions actionnelles permettant de décrire la gestion enseignante. Ces dimensions sont postulées comme génériques de l'activité de l'enseignant et, à ce titre, devaient permettre, potentiellement, de décrire les phénomènes didactiques quelqu'en soit l'objet. Différents chercheurs ont testé ces catégories sur différents objets disciplinaires (voir notamment Cordoba, 2009; Quilio, 2012; Sensevy, 2001) afin d'en examiner les limites et les possibles. 
Les principales dimensions actionnelles sont les suivantes. En revisitant la notion de milieu (au sens de Brousseau, 1990), travaillée en didactique des mathématiques, les comparatistes en didactique ont proposé le concept de mesogenèse plus propre à décrire une dynamique d'évolution de ce milieu. La mesogenèse relève des objets de l'interaction enseignant-élèves tels qu'ils évoluent et s'organisent en une trame de significations partagées ou non. Avec un point de vue plus directement tourné vers les acteurs, un second concept, la topogenèse, permet une description des prises en charge respectives entre enseignant et élèves au fil de leur interaction: qui fait quoi et dans quelle posture, qui amène les objets de la mesogenèse, les définit, les «fait vivre» dans la classe. Enfin, l'analyse de la chronogenèse du point de vue des savoirs en jeu permet une description de l'avancement de l'enseignement/apprentissage au fil de l'interaction Une théorisation en termes de tâches précises relevant de l'enseignant versus des élèves reste sans doute à affiner. En l'état, du point de vue de l'hétérogénéité, les travaux permettent de décrire ce que les didacticiens comparatistes nomment la "construction de milieux différentiels» (Ligozat \& Leutenegger, 2008) qui s'inscrit à la suite des travaux sur le contrat didactique différentiel (Leutenegger \& Schubauer-Leoni, 2002; Schubauer-Leoni, 1987, 1996). L'intérêt de cette notion réside dans la possibilité de décrire, du point de vue des objets, un processus qui peut s'avérer différent selon les élèves.

Chaque objet de la mesogenèse, fut-elle différentielle, est potentiellement un moyen de faire progresser la chronogenèse en fonction des rapports que les sujets établissent à celle-ci. L'observation des objets qui, de fait, construit une référence (évolutive) entre les acteurs, enseignant et élèves, constitue une entrée prioritaire. Mais la dissymétrie des positions d'enseignant et d'élève (par définition) détermine un partage des responsabilités et des tâches, qui nous amène à analyser la topogenèse. Nous examinerons ici des postures contrastées d'élèves dans leur rapport aux objets. Ce que nous nommerons une topogenèse différentielle pour marquer non seulement la différence de position «verticale» entre enseignant et élèves, mais également celle plus "horizontale» entre pairs.

Ces différents registres jouent un rôle central dans les analyses. Nous présenterons maintenant les enjeux didactiques, avec une brève analyse des tâches soumises aux élèves et aux enseignantes, pour chacun des deux terrains d'observation puis nous exposerons les résultats empiriques de chacune.

\section{Enjeux didactiques en classe de $3 P$}

Une activité concernant le nombre, lié à l'énumération de collections équipotentes est au cœur de cette observation. Ce type d'activité repose sur des travaux anciens déjà (notamment Meljac, 1979, dans la mouvance des études piagétiennes) concernant le dénombrement spontané chez l'enfant. Ces travaux repris en didactique par El Bouazzaoui (1982) et par Briand (1999), portent sur diffé- 
rentes situations dont le principe commun est le suivant: faire correspondre, numériquement, deux collections distantes spatialement. Par exemple, il s'agit, pour de jeunes enfants, d'aller chercher ce qu'il faut de robes, pas plus, pas moins, pour habiller quatre, six ou neuf poupées, la distance entre les deux collections (robes et poupées) étant considérée comme une variable de situation puisqu'elle détermine des contraintes différentes: dans le cas d'une coprésence des collections, une correspondance terme à terme entre chaque poupée et chaque robe est possible et ne contraint pas, comme lorsque les collections sont distantes, une énumération et un comptage systématique pour réussir la correspondance.

À partir de la situation des "poupées», et plus généralement de situations dérivées, l'école élémentaire, notamment en Suisse Romande, préconise des activités de ce type en introduction au concept de nombre auprès des jeunes élèves. C'est ainsi qu'en $3 \mathrm{P}$ et $4 \mathrm{P}$, des fiches, respectivement intitulées "Les cousins» et "La cible», supports d'activités "phares» selon les auteurs, consistent à dénombrer et à aller chercher, en un nombre restreint de trajets, voire un seul, une quantité de vignettes (d'une seule ou de deux couleurs selon la fiche) permettant de recouvrir un nombre équivalent de cases formant l'image d'un personnage ou d'une cible carrée. Faute d'une proximité entre la fiche et les vignettes, l'élève ne peut effectuer une correspondance terme à terme. Une vérification matérielle (rétroaction) permet toutefois à l'élève de savoir si une erreur est intervenue, mais, cas échéant, à lui de trouver laquelle.

Dans le cas particulier de cette observation, et bien qu'appartenant aux moyens d'enseignement de 4P-H, «La cible» (Fig. 1) est choisie par l'enseignante de cette classe de $3 \mathrm{P}-\mathrm{H}$; elle y apporte toutefois des modifications visant à rendre le problème compatible avec les exigences de ce degré.
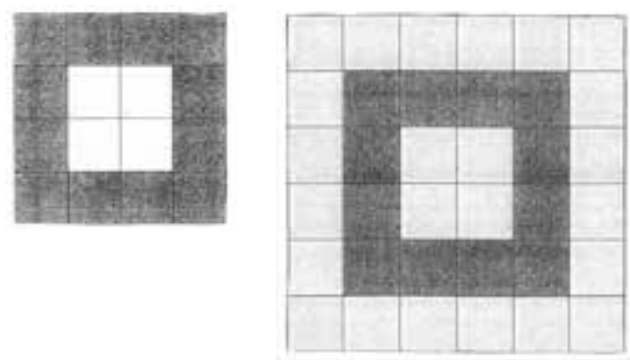

Figure 1: Cibles 1 et 2

Il s'agit de recouvrir les cases (jaunes et rouges) de chacune des deux "cibles». Pour la Cible 1, il s'agit de se doter de deux collections, l'une de 4 jetons jaunes et l'autre de 12 jetons rouges et, pour la Cible 2, de deux collections de 24 jaunes et 12 rouges (ou trois collections correspondant aux rangs concentriques: 4 jaunes, 12 rouges, 20 jaunes). La Cible 1 est réputée «plus simple» que la Cible 2 puisqu'elle suppose des quantités plus réduites, la Cible 2 se complexifiant par 
la présence de deux sous-collections de cases jaunes. Seuls l'énumération et le dénombrement de chacune des collections et la mémoire du cardinal permet de réaliser la correspondance et de recouvrir les cases jaunes et rouges.

\section{Enjeux didactiques en classe de 4P}

Il s'agit d'une séquence de longue durée (plusieurs mois) visant l'apprentissage de la soustraction. Une ingénierie didactique dont l'origine, dans les années 1980, revient aux équipes de G. Brousseau dans le cadre du COREM ${ }^{2}$, est au cour de la présente contribution. Cette situation a été reprise dès 2009 par l'un d'entre nous (Quilio 2009/2010) afin d'en affiner les caractéristiques, notamment de gestion par l'enseignant.

L'idée fondatrice de cette ingénierie est la suivante: l'enseignement repose sur le savoir antérieur des élèves, c'est-à-dire l'addition, pour construire les différents sens de la soustraction, repérer et résoudre les problèmes qui peuvent se résoudre avec une soustraction. Ainsi dans la genèse de l'apprentissage envisagé, deux rôles sont assignés à l'addition: elle joue un rôle dans la construction des sens de l'opération de soustraction et elle fonde la recherche d'un moyen économique de calcul des différences, une technique.

Dans le cas des observations présentées ici, et qui se situent en fin de séquence (leçon 12 et 13 de la progression qui en comporte 15), voyons quels sont les enjeux mathématiques. Il s'agit d'introduire aux premières productions de la technique opératoire. Avant ces leçons, les stratégies de calcul des élèves pour trouver la différence dans un problème de soustraction peuvent être diverses. Seule la vérification par addition est commune. Il s'agit maintenant de faire formuler, reconnaitre, identifier par tous, les stratégies qu'ils ont produites pour déterminer les différences, et de mettre en place une technique économique. L'algorithme de la soustraction n'intervient donc en aucune façon comme objectif, mais plutôt comme un outil permettant le calcul une fois le problème à résoudre compris et posé. À noter que des évaluations (prévues par le dispositif séquentiel) interviennent, dès le début de la séquence, à intervalles réguliers et constituent une forme de rétroaction à l'enseignant et aux élèves sur ce qui a été appris ou non.

Centrons-nous sur ces leçons 12 et 13, un peu avant que l'algorithme de soustraction soit travaillé en tant qu'outil. Lors de la leçon 12, il s'agit de travailler différentes stratégies de résolution de problèmes soustractifs. Ceux-ci sont résolus, fictivement, par des «schtroumpfs». Le document de référence précise que:

Pour prendre du recul le maittre introduit dans la classe des personnages extérieurs dont on peut débattre des stratégies de façon neutre. Ce ne sont pas des élèves de la classe. On est plus à l'aise pour commenter ce qu'ils font. (p. 64) 
Il s'agit, par ce subterfuge, de travailler le statut des nombres à faire intervenir dans la résolution et à limiter les erreurs de calcul. En effet, l'algorithme n'intervient pas encore et les élèves résolvent le problème par essais-erreurs en procédant «en ligne» ou par tout autre moyen permettant de poser le problème et de savoir quel nombre soustraire ou additionner à un autre. Par exemple, le problème suivant fait partie de la leçon 12:

Ce matin le facteur avait 93 lettres à distribuer. Il a déjà distribué 56 lettres. Combien en a-t-il dans sa sacoche maintenant?

Dans la leçon 13 qui sera analysée plus loin, les deux problèmes posés sont les suivants:

Problème 1: Dans les deux classes de 3P-4P, il y a 84 élèves. Nous avons compté 38 filles. Combien y a-t-il de garçons?

Problème 2: Dans un troupeau il y a 71 moutons, des noirs et des blancs. Il y a 32 moutons noirs. Combien y a-t-il de moutons blancs?

Ce type de problème est censé amener la classe d'une part à lui donner sens (rapports entre les quantités en présence, les parties, le tout). Au plan calculatoire, les caractéristiques numériques sont pensées pour obliger une réflexion liée à la numération de position précisément ici avec la question de la retenue.

Deux propositions attribuées aux «schtroumpfs» pour le problème 1 seront décisives dans les interactions relevées lors de la mise en œuvre de cette leçon:

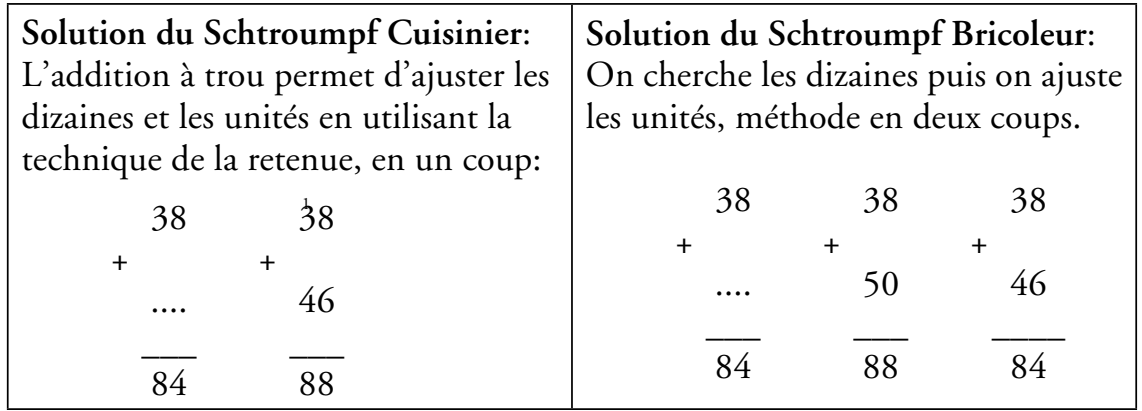

\section{Quelques enjeux didactiques comparés}

Les contenus d'apprentissages visés par les deux situations sont différents: en 3P le dénombrement de collections et l'usage de la notation chiffrée, en 4P l'identification de stratégies liées aux propriétés des nombres pour déterminer la différence dans des problèmes soustractifs. Du point de vue de l'organisation 
des savoirs, la connexité entre les fiches «Cibles» et la précédente («Les cousins») existe bien, mais les deux fiches peuvent fonctionner indépendamment. Dans le cas de la soustraction, au contraire, l'organisation du «savoir soustraire» porte sur une séquence de plusieurs mois. Les connexités sont construites et, du point de vue des variables numériques, chaque problème de chaque leçon est soigneusement élaboré en vue de certains effets didactiques. Les situations ont néanmoins des enjeux didactiques communs. Elles imposent aux enseignants de gérer le passage d'interactions individuelles avec des objets choisis dans le milieu, à la construction d'une réponse stratégique partagée par toute la classe. Observons maintenant des épisodes de cette réalisation.

\section{En classe de 3P}

Le dispositif d'observation comprend une leçon de 60 minutes et deux entretiens avec l'enseignante, permettant de considérer son point de vue sur la leçon, avant et après.

Après une brève description de la structure de la séance, nous ferons état de quelques résultats majeurs concernant l'observation de deux élèves, respectivement déclarés «fort» (Vitorio) et «faible» (Jérémie) en mathématiques, pour décrire le fonctionnement d'un contrat didactique différentiel.

Le tableau synoptique de la séance (voir Annexe 1) indique qu'après une introduction où l'enseignante rappelle collectivement les activités précédentes, elle présente la tâche du jour. Puis, une phase de travail individuel, jusqu'à la récréation, consiste à traiter le problème de la Cible 1 puis celui de la Cible 2. L'enseignante gère le passage d'un problème à l'autre pour chaque élève: la Cible 2 n'est traitée que lorsque la Cible 1 a trouvé une solution. Après la récréation, les élèves sont réunis pour une "mise en commun». Enfin, la séance se termine par l'annonce d'une évaluation quelques jours plus tard.

\section{Au plan de la mesogenèse}

De fait, les deux élèves n'ont pas été confrontés aux mêmes tâches puisque Vitorio résout les deux problèmes alors que Jérémie ne termine que le premier, l'enseignante lui évitant la confrontation avec le second. Cela constitue-t-il pour Jérémie, une occasion de moins d'apprendre? Plus tard, une évaluation confirmera que Jérémie n'a pas progressé dans ce type d'apprentissage. Alors que Vitorio a appris en tout cas à traiter ce type de problème, Jérémie n'a fait qu'appliquer ce que l'enseignante lui a suggéré: compter les vignettes et noter le cardinal.

Pour ce qui concerne les phases de travail individuel, l'analyse montre que Vitorio et Jérémie sont confrontés à un milieu mathématique différent (mesogenèse différentielle): le milieu reste entièrement numérique pour Jérémie (il n'est confronté qu'à un problème de comptage instauré par l'enseignante) 
alors que pour Vitorio, le milieu est surtout lié au problème qu'il se pose à lui-même: comment garder en mémoire les quantités de jaunes et de rouges afin de répondre à la contrainte de n'effectuer qu'un seul voyage? Ce milieu est donc lié à la notation des quantités.

A noter que les deux problèmes sont repris lors de la «mise en commun». Dès lors, une question se pose: que peut dire Jérémie (ou d'autres élèves dans le même cas) de ce problème dont il n'a pas connaissance? Il est d'emblée disqualifié lors de ce débat collectif et du reste n'y participe pas. Au contraire de Vitorio qui peut faire état de sa façon de traiter le problème, particulièrement ses notations, pour mémoire, des nombres de vignettes jaunes et rouges.

\section{Au plan de la topogenèse}

Le découpage de la tâche de Jérémie est pris en charge par l'enseignante, au contraire de ce qui se passe pour Vitorio. Pour Jérémie, elle insiste du reste pour qu'il trouve un moyen, autre que le comptage, pour garder en mémoire les quantités de jetons nécessaires, ce faisant, c'est elle qui suggère la notation. L'analyse montre, notamment grâce aux entretiens avec l'enseignante, qu'elle attend expressément des élèves qu'ils recourent à la notation, comme le fait de lui-même Vitorio (il est le seul de la classe à mobiliser ce procédé) et comme elle le suggère aux autres et notamment à Jérémie.

Nonobstant les attentes différentielles de l'enseignante concernant ces deux élèves (les entretiens le montrent), des différences substantielles dans l'action enseignante auprès d'eux ont été observées. Vitorio, dans un premier temps, agit seul pour résoudre le problème: l'enseignante n'intervient pas. Au contraire, l'enseignante intervient à plusieurs reprises pour réorienter l'action de Jérémie. En ce qui concerne les types d'action, on observe, de la part de l'enseignante, relativement moins de gestes visant à évaluer la pertinence des actions de Vitorio que celle des actions de Jérémie. Pour ce dernier, à chaque passage devant l'enseignante, une évaluation se fait jour.

L'observation des deux élèves contrastés met ainsi en évidence le fonctionnement d'un contrat didactique différentiel. Les conduites de Jérémie répondent aux attentes supposées de l'enseignante, mais ne parviennent pas à tisser un référentiel commun avec le reste de la classe, puisque l'usage d'une notation ne répond pas, au moins pour cet élève, à une fonction pour le problème posé, mais constitue plutôt une réponse sociale à la demande de l'enseignante. Le constat principal est le suivant: l'enseignante opère par différenciation auprès de cette classe hétérogène. Une gestion parfaitement conforme à ce qui est attendu institutionnellement: la différenciation n'est-elle pas réputée porteuse d'une meilleure adaptation de l'enseignement à chaque cas d'élève et donc amener de meilleurs résultats quant aux apprentissages? Or, le constat est bien différent: cette gestion individuelle des tâches entraîne un certain nombre de conséquences qui ne font, au final, que creuser l'écart entre les élèves. Nous souhaitons interroger cette pétition de principe d'une différenciation nécessaire et nous le ferons 
maintenant à la lumière d'une étude comparative (en 4P) qui offre d'autres registres de gestion par l'enseignante. Une différenciation là aussi, mais une différenciation portée par une gestion collective des problèmes mathématiques posés.

\section{En classe de 4P}

Testée dans plusieurs classes marseillaises, la séquence «soustraction» a été diffusée dans le cadre du Réseau Maison des Petits à Genève ${ }^{3}$ et travaillée dans deux classes de 4P. La grande majorité des leçons a été filmée et toutes les traces d'écriture provenant des élèves et des enseignantes ont été collectées de même que les préparations écrites des enseignantes ${ }^{4}$. Dans l'une des classes (double degré 3P-4P), les 12 élèves de $4 \mathrm{P}$ ont été observés à la fois dans le cadre des échanges collectifs et dans le cadre de la résolution individuelle des problèmes soustractifs. Pour cette contribution, nous nous focaliserons sur trois élèves considérés comme "faibles» au début de la séquence par leur enseignante, en examinant ce qu'il advient de leurs apprentissages en matière de soustraction en fin de séquence.

Les élèves sont confrontés à des problèmes soustractifs depuis plusieurs semaines selon la progression prévue par l'ingénierie. Or, dès les premières leçons, trois élèves (MAE, TOM et LAR) rencontrent diverses difficultés - plus que les autres élèves - de compréhension du sens des problèmes. Où est le tout? Quelles sont les parties? Que retirer à quoi? Peut-on additionner pour trouver la solution? Telles sont les questions que rencontrent tous les élèves, mais ces trois-là ne parviennent que difficilement à y répondre et à résoudre les problèmes. Nous examinerons ce qu'il advient de ces élèves lorsque la situation d'ingénierie arrive à terme, aux leçons 12 et 13 .

Lors de la leçon 12, l'enseignante introduit une nouveauté - prévue par l'ingénierie - les élèves débattent désormais à partir de solutions, fictivement trouvées par des «schtroumpfs». Lors de cette leçon, LAR, TOM et MAE rencontrent un obstacle majeur (mais les autres élèves également) relatif au choix numérique des problèmes: comment, en effet, soustraire par exemple 56 de 93 en procédant à des soustractions partielles successives (dizaines et unités) puisque l'algorithme n'est pas encore à disposition?

$(\ldots)$

Ens: «qu'est-ce qu'on devait faire, nonante-trois moins cinquante-six, LAR me disait mais je ne peux pas enlever six à trois, elle a entièrement raison, donc puisque je suis bloquée, je ne peux pas enlever un plus grand nombre à un plus petit nombre».

(...) 
L'ensemble de la leçon, centrée sur deux problèmes de ce type, tourne autour de cette question du traitement numérique. La pose en ligne de l'opération sur la base de l'énoncé du problème ne présente, à ce stade, plus d'obstacle pour personne, pas même pour LAR, MAE et TOM qui, antérieurement achoppaient là dessus. Témoins les premières évaluations réalisées par l'enseignante.

$\mathrm{Au}$ terme de la leçon 12, marquée par des débats collectifs à propos des solutions individuelles, une méthode commune a été construite: par exemple pour $84-18$, enlever d'abord les dizaines (ici 10 de $84=>74$ ) puis les unités (74 - 8 = 66), c'est-à-dire en décomposant, comme le relève l'enseignante:

"(...) on peut enlever par petits groupes hein, comme dit CHL, quand je suis à septante-quatre, j'enlève quatre ça fait septante, j'enlève encore quatre et ça me fait soixante-six hein, vous pouvez enlever soit dix par dix soit en prenant les unités $(\ldots)$ »

Voyons maintenant une analyse plus fine de la leçon 13 , pour les trois élèves considérés les plus en difficulté par leur enseignante au début de la séquence. Comme pour la leçon de 3P, nous examinerons ce qu'il advient de la mesogenèse et de la topogenèse différentielles. In fine, nous en tirerons un certain nombre de conséquences au plan de la chronogenèse pour le collectif de la classe.

\section{Au plan de la mesogenèse}

Face au premier problème du jour, chacun des trois élèves a sa technique propre (techniques qu'ils appliqueront aussi aux problèmes suivants) que nous reconstituons à l'aide des écrits recueillis:

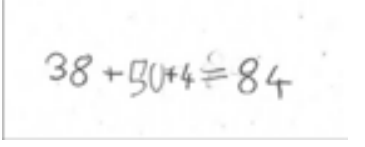

TOM

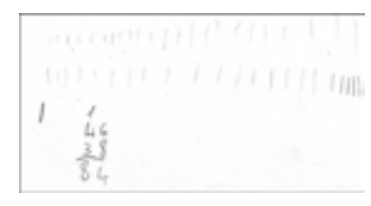

MAE

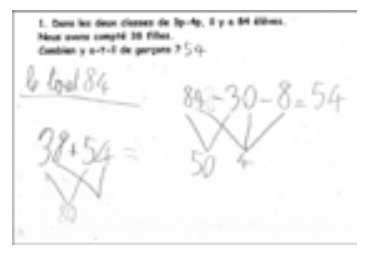

LAR

TOM pose une addition en ligne (erronée); MAE en pose une en colonne après avoir noté sous forme de bâtons la quantité à soustraire (solution 46 correcte); LAR, quant à elle, après avoir noté le tout (84), tente de mettre en œuvre une technique apprise initialement pour les additions en ligne, mais qui ne marche pas très bien - évidemment - pour des soustractions de ce type et lorsque figurent, comme ici, plusieurs «membres» à la soustraction ${ }^{5}$; on peut reconstituer son action de la façon suivante: $80-30=50 ; 4-0-8=4$ (on retrouve ici l'obstacle de la leçon 12: comment enlever un plus grand nombre à un plus petit?); $50+4$ 
= 54. Mais comment interpréter l'addition qui suit: $38+54=>80$ ? S'agirait-il d'une démarche de preuve non aboutie? Car $30+50=80$ en effet. Si elle avait poursuivi, elle aurait peut-être constaté que $8+4$ font 12 et que $80+12$ ne font pas 84 . Mais elle n'en a pas le temps: l'enseignante appelle les derniers élèves à se rassembler pour un débat autour des solutions proposées par «les schtroumpfs».

À noter que cette hétérogénéité au niveau des techniques de résolution (propres à d'autres élèves également) est parfaitement admise par l'enseignante et à aucun moment elle n'intervient à ce sujet. Un milieu différentiel est donc toléré, voire encouragé, puisque l'enseignante, avant l'examen des solutions des schtroumpfs, note au tableau noir les opérations posées par les élèves (sans les solutions).

\section{Au plan de la topogenèse}

Nous examinerons sur la base d'un tableau synoptique de la séance (voir annexe 2), la place de ces trois élèves dans la séance. Puis nous commenterons quelques événements significatifs (interactions enseignante/élèves) du fonctionnement du contrat didactique différentiel.

Le tableau synoptique indique deux phases correspondant aux deux problèmes $^{6}$. Le déroulement en quatre étapes est semblable: 1) l'énoncé du problème est lu par deux élèves; 2) le problème est traité individuellement; 3 ) en collectif, le problème est posé (sans la solution); 4) chacune des solutions des schtroumpfs est examinée, débattue.

Les trois élèves sont fortement présents lors des débats collectifs. Pour le problème 1, l'enseignante sollicite chacun d'eux à un titre ou l'autre: MAE pour poser le problème sous forme d'addition et pour revenir, plus tard, sur sa solution (correcte); TOM sur la différence entre 84 (nombre total d'élèves) et 88 (nombre correspondant à la solution du schtroumpf bricoleur); LAR du point de vue de la relation de sa solution avec celle du schtroumpf cuisinier. Pour le problème 2 , MAE n'est pas sollicitée. TOM, qui, lui aussi, a résolu celui-ci, est sollicité pour statuer sur les solutions des schtroumpfs. À noter qu'à cette occasion, il souffle la réponse attendue à un autre élève (Bar) considéré, d'habitude, comme un élève moyen. LAR est sollicitée pour comparer sa solution (erronée) avec celle du schtroumpf musicien. Comme pour le problème 1, la pose est correcte, mais une erreur de calcul est intervenue. En l'état des analyses, notons que ces trois élèves ne se distinguent plus fondamentalement des autres: au terme de cette leçon 13 , ils parviennent tous à poser le problème en identifiant les rapports entre les nombres (le tout et les parties) et deux sur les trois calculent correctement à partir de cette pose. Ceci constitue un résultat tout à fait remarquable puisque les premières leçons indiquent au contraire des difficultés récurrentes pour ces trois élèves. L'écart ne s'est donc en tout cas pas creusé avec le reste de la classe, comme c'était le cas de la leçon de 3P.

D'élèves «en difficulté» en début de séquence à élèves «moyens» au terme de cette séquence, ces élèves changent-ils quelque peu de statut? 
Pour tenter d'expliquer cette évolution, voyons maintenant deux extraits emblématiques du type d'interaction instaurée par l'enseignante pour la gestion collective de la situation. Celle-ci intègre les trois élèves.

Extrait 1: échange avec MAE et la classe sur le pb. 1

\begin{tabular}{|c|c|c|}
\hline $\begin{array}{l}13^{\prime} 54 \\
\text { à } \\
16^{\prime} 01\end{array}$ & $\begin{array}{l}\text { ** (pb. 1: solution Schtroumpf cuisinier: } 38+46 \\
\text { en colonne) } \\
\text { (...) } \\
\text { Ens: alors est ce qu'on peut// prouver qu'il a } \\
\text { raison/ MAE } \\
\text { MAE: on fait huit plus six ça nous donne quatorze? } \\
\text { Ens: quatorze (écrit } 14 \text { au TN sous } 38+46 \text { ) } \\
\text { MAE: tu mets le un dans les dizaines? } \\
\text { Ens alors pourquoi je mets le un dans les dizaines } \\
\text { (efface le 1 et l'écrit au dessus du } 3 \text { de 38) } \\
\text { MAE: parce que celui qui est devant le quatre c'est } \\
\text { une dizaine? } \\
\text { Ens: oui/ mais pourquoi je le mets? } \\
\text { MAE: parce que si on met/ heu je sais pas? } \\
\text { Ens: tu sais pas/ Mar / pourquoi on l'a vu l'autre } \\
\text { jour quand on fait des additions/ que j'ai une petite } \\
\text { retenue/ mais pourquoi/ ça représente quoi? } \\
\text { Elg: ça représente la dizaine? } \\
\text { Ens: Mar? } \\
\text { Mar: ca représente la dizaine parce que heu heu } \\
\text { pour faire quatorze on doit faire dix plus quatre et } \\
\text { le dix tu vas le mettre dans la colonne des dizaines } \\
\text { Ens: voilà// heu MAE/ t'as bien fait huit plus six? } \\
\text { MAE: (oui de la tête) } \\
\text { (...) }\end{array}$ & $\begin{array}{l}\text { Ecritures } \\
\text { au TN }\end{array}$ \\
\hline
\end{tabular}

Le débat porte sur l'algorithme d'addition (à retenues). MAE est en difficulté pour expliquer le statut de la retenue. A noter que MAE, dès le début de la séquence est une élève qui tente de résoudre tous les problèmes à l'aide d'un algorithme (dans quelles conditions l'a-t-elle appris?), mais faute de donner du sens aux relations entre les nombres dans les problèmes, elle ne parvient pas à des solutions valides: elle semble appliquer l'algorithme comme «un truc». L'enseignante, dans les phases précédentes lui a interdit de faire usage de l'algorithme, au profit de stratégies de notation des quantités incriminées. Comme la plupart des élèves, elle a appris à noter des bâtons, à se représenter les quantités par des cubes ou autres. Or, à cette étape, elle est l'une des seules élèves à noter encore des bâtons sur sa feuille correspondant aux nombres concernés: apparemment ceci l'aide à passer à une écriture et l'enseignante laisse faire malgré le fait que 
cette notation n'est plus en adéquation avec ce qui est attendu en cette fin de séquence. Mais ce milieu (différentiel) permet tout au moins le débat collectif et la participation de MAE à celui-ci. MAE est installée désormais dans une position d'élève qui contribue au débat et du reste sa solution est reconnue comme correcte.

Extrait 2: échange avec TOM et la classe sur le pb. 1

\begin{tabular}{|c|c|c|}
\hline $\begin{array}{l}12^{\prime} 03 \\
\text { à } \\
13 ’ 11 \\
16^{\prime} 56 \\
\text { à } \\
18^{\prime} 05\end{array}$ & $\begin{array}{l}\text { *(solution du Schtroumpf bricoleur } 38+50 \text { en } \\
\text { colonne = } 88 \text { est au TN) } \\
\text { Résumé: TOM intervient sur la différence entre } \\
84 \text { (nombre total d'élèves) et } 88 \text { (nombre corres- } \\
\text { pondant à la solution du schtroumpf bricoleur). } \\
\text { Discussion: faut-il } 4 \text { de plus ou } 4 \text { de moins? } \\
\text { *** (sur la base des deux solutions des } \\
\text { schtroumpfs notées au TN } 38+50=88 \text { et } 38+ \\
46=84) \\
\text { (...) } \\
\text { Ens: alors pour revenir à la question d'avant TOM/ } \\
\text { quand tu m'as dit mais/ y en a quatre de trop/ } \\
\text { donc j'vais en enlever quatre// y a combien de } \\
\text { différence entre cinquante et quarante-six? } \\
\text { TOM: heuu heu quatre } \\
\text { Ens: ici? (montre } 50 \text { et } 46 \text { ) } \\
\text { TOM: oui? } \\
\text { Ens: le schtroumpf a trouvé cinquante garçons// et } \\
\text { tu m'as dit mais y en a quatre de trop// alors qu'est } \\
\text { ce que tu fais pour en avoir quatre heu en moins } \\
\text { de garçons// qu'est ce qui s'est passé entre là et là? } \\
\text { (montre les deux solutions de schtroumpfs) } \\
\text { TOM: (xxx) } \\
\text { Ens: t'avais raison quand t'as dit ha mais à quatre- } \\
\text { vingt-huit y en a quatre de trop parce que nous on } \\
\text { en veut pas quatre-vingt-huit on en veut quatre- } \\
\text { vingt-quatre/ donc qu'est ce que je vais faire à } \\
\text { partir de cinquante// si cinquante c'est trop// tu } \\
\text { comptes à rebours et t'enlèves combien? } \\
\text { TOM: quatre? } \\
\text { Ens: voilà/ on veut quatre garçons de moins/ donc } \\
\text { si t'en trouves cinquante// j'en enlève quatre/ } \\
\text { cinquante moins quatre// chuut? } \\
\text { TOM: quarante-six? } \\
\text { (...) }\end{array}$ & $\begin{array}{l}\text { Ecritures au } \\
\text { TN }\end{array}$ \\
\hline
\end{tabular}


L'enseignante procède en deux temps en s'appuyant sur les solutions des schtroumpfs: premièrement, avec la solution du schtroumpf bricoleur, TOM n'a considéré que le tout et la différence entre 88 et 84 . Or, la solution du problème ne porte pas sur le tout, mais sur l'une des parties, c'est-à-dire le nombre de garçons. Au regard des solutions des schtroumpfs, l'enseignante focalise l'attention de TOM sur cette partie (46 et 50). De fait, TOM, sur sa feuille, a noté $50+4$ : il a donc en effet ajouté 4 au lieu d'enlever. L'intervention collective de l'enseignante permet à TOM de comprendre son erreur et, du reste, il résoudra le second problème sans difficulté.

Que retenir de l'analyse de ces extraits significatifs? Notons tout d'abord que les élèves agissent sur des milieux différentiels (les notations, les discours sur ces notations) et que l'enseignante les laissent faire: elle n'intervient pas à ce niveau pour tenter d'unifier le milieu. Chacun peut agir sur le problème à partir d'une soustraction ou d'une addition (en ligne ou en colonne) ou d'une autre technique encore. Mais dans tous les cas, le traitement est collectif: les solutions des schtroumpfs d'abord, des élèves ensuite, sont diffusées et débattues collectivement. La construction effective chez tous les élèves de l'addition comme élément fondateur de résolution de ce type de problèmes met l'enseignante en disposition d'enseigner même aux élèves en difficulté (la force de l'usage du nombre pour MAE, le travail de dénotation des écritures mathématiques pour TOM), tout en faisant avancer collectivement la construction de la connaissance mathématique car tous sont confrontés, à des nivaux sans doutes multiples, au problème dont l'algorithme sera la solution.

\section{Brève conclusion}

Les analyses montrent que les deux enseignantes traitent de façon opposée l'hétérogénéité des rapports aux tâches à accomplir dans les situations. Dans la classe de 3P, les difficultés rencontrées par Jérémie sont prises en compte de façon individuelle, mais il ne parvient pas à entrer dans l'enjeu de la situation proposée. Dans la classe de 4P, le traitement collectif des stratégies des élèves dits "en difficulté» sont discutées et travaillées collectivement car elles permettent à l'enseignante «d'organiser une rencontre collective» avec la question dont le savoir mathématique est une réponse. D’un point de vue mesogénétique, ce qui est pris en charge explicitement par les situations oriente de façon décisive les options topogénétiques possibles face à l'hétérogénéité des réponses des élèves. Ainsi, dans le cas de la soustraction, la situation envisage d'emblée les cas de figure (les stratégies des différents schtroumpfs) qui permettent à l'enseignante d'opérer un «bon réglage» entre les options topogénétiques (ce qui est à la charge de la situation, des élèves, ce que l'enseignant doit enseigner) et les ressources du milieu. Il ressort de nos observations que ce type de «réglage» conditionne vraisemblablement à la fois l'avancée du temps d'apprentissage mais aussi la 
valence chronogène de l'hétérogénéité pour l'avancée du temps didactique de la classe. Un élargissement à l'analyse d'autres classes seraient néanmoins bienvenu, voire nécessaire, pour corroborer ces résultats.

\section{Notes}

1 Voir notamment les travaux de Bronckart (2004) qui montrent l'intérêt d'une distinction entre activité, action de la personne singulière et action collective, en remontant aux différentes significations des concepts d'activité et d'action.

2 Centre pour l'Observation et la Recherche sur l'Enseignement des Mathématiques.

3 Les participants du «Réseau Maison des Petits» à Genève et ceux du LéA Saint Charles (Lieu d'éducation Associé à l'Institut Français de l'Éducation) à Marseille collaborent depuis plusieurs années et une convention de recherche a été signée par les institutions en 2011. Ces accords de collaboration sont l'occasion de tester différentes situations didactiques de part et d'autres, la séquence sur la soustraction en fait partie, comme a été travaillée aussi bien à Marseille qu'à Genève une autre situation, nommée "Jeu du Trésor» destinée aux élèves de l'école enfantine/maternelle qui a fait l'objet de plusieurs publications (voir notamment Forget \& Schubauer-Leoni, 2008; Leutenegger \& Quilio, en préparation; Schubauer-Leoni, Leutenegger \& Forget, 2007).

4 Nous ne pouvons nous appuyer ici sur des entretiens. Néanmoins, les préparations écrites de l'enseignante sont utiles pour cerner son projet.

5 À noter la pose de $84-30-8$, avec deux soustractions successives, non admise en principe. Cette pose est tolérée ici au regard de la technique de décrémentations successives apprise au terme de la leçon 12 .

6 Problèmes 1 et 2: voir ci-dessus «enjeux didactiques en classe de 4P».

\section{Références bibliographiques}

Briand, J. (1999). Contribution à la réorganisation des savoirs pré-numériques et numériques. Etude et réalisation d'une situation d'enseignement de l'énumération dans le domaine pré-numérique. Recherches en Didactique des Mathématiques, 19/1, 41-75.

Bronckart, J.-P. (Ed.). (2004). Agir et discours en situation de travail (Cahiers de la Section des sciences de l'éducation $\left.\mathrm{N}^{\circ} 103\right)$. Genève: Université de Genève.

Brousseau, G. (1990). Le contrat didactique: le milieu. Recherche en didactique des mathématiques, 9/3, 309-336.

Cordoba, A. (2009). Référentiels des enseignants et construction de la référence dans l'action conjointe maitre-élèves en éducation physique: étude comparative de l'action didactique des maitres généraliste et spécialiste. Actes du ler colloque international de l'ARCD. Où va la didactique comparée? Didactiques disciplinaires et approches comparatistes des pratiques d'enseignement et d'apprentissage. Édition: Université de Genève FPSE-SSED \& ARCD. [CD-Rom]

El Bouazzaoui, H. (1982). Etudes de situations scolaires des premiers enseignements du nombre et de la numération. Thèse Université Bordeaux I.

Forget, A. \& Schubauer-Leoni, M.-L. (2008). Inventer un code de désignation d'objets au début de la forme scolaire: des productions personnelles à la convention collective. In L. Filliettaz \& M.-L. Schubauer-Leoni (Éd.), Processus interactionnels et situations éducatives (Coll. Raisons éducatives, pp. 183-204). Paris/Bruxelles: De Boeck.

Leutenegger, F. (2009). Le temps d'instruire: approche clinique et expérimentale du didactique ordinaire en mathématique (Coll. Exploration). Berne: Peter Lang.

Leutenegger, F. \& Schubauer-Leoni, M.-L. (2002). Les élèves et leur rapport au contrat didactique: une perspective de didactique comparée. In A. Terrisse (Éd.), Les didactiques scientifiques et technologiques. Les dossiers des sciences de l'éducation, 8, 73-86. 
Leutenegger, F. \& Quilio, S. (en préparation). Pratiques et développement professionnel au cycle 1 . Dispositif d'analyse conjointe entre chercheurs et praticiens.

Ligozat, F. \& Leutenegger, F. (2008). Construction de la référence et milieux différentiels dans l'action conjointe du professeur et des élèves: le cas d'un problème d'agrandissement de distances. Recherches en Didactique des Mathématiques, 28 (3), 319-378.

Meljac, C. (1979). Décrire, agir, compter. Paris: PUF.

Mercier, A., Schubauer-Leoni, M.-L. \& Sensevy, G. (Éd.). (2002). Vers une didactique comparée. Revue Française de pédagogie, 141. Paris: INRP.

Quilio, S. (2009/2010). La soustraction au CE1. Document de recherche non publié, Institut Français de l'Éducation, École normale supérieure de Lyon.

Quilio, S. (2012). Une forme caractéristique de condition de l'étude dans la réalisation d'une ingénierie didactique en mathématiques à l'École Primaire. Éducation \& Didactique, 6 (2), 9-26.

Schubauer-Leoni, M.-L. (1987). Le contrat didactique: un cadre interprétatif pour comprendre les savoirs manifestés par les élèves en mathématiques. European Journal of Psychology of Education, 1 (2), 139-153.

Schubauer-Leoni, M.-L. (1996). Étude du contrat didactique pour des élèves en difficulté en mathématiques. In C. Raisky \& M. Caillot (Éd.), Au-delà des didactiques, le didactique (pp. 159-189). Bruxelles: De Boeck Université.

Schubauer-Leoni, M.-L, Leutenegger, F. \& Forget, A. (2007). Premières expériences cruciales de la forme scolaire: l'accès aux pratiques de fabrication de traces scripturales convenues. Education \& Didactique, 1 (2), 9-35.

Sensevy, G., Mercier, A. \& Schubauer-Leoni, M.-L. (2000). Vers un modèle de l'action didactique du professeur à propos de la course à 20. Recherches en didactique des mathématiques 20(3), 263-304.

Sensevy, G. (2001). Théories de l'action et action du professeur. In J.-M. Baudouin \& J. Friedrich (Éd.), Théories de l'action en éducation (Coll. Raisons éducatives, pp. 203-224). Paris/Bruxelles: De Boeck.

Sensevy, G. \& Mercier, A. (Éd.). (2007). Agir ensemble: l'action didactique conjointe du professeur et des élèves. Rennes: Presses Universitaires de Rennes.

Mots-clés: Contrat didactique différentiel, action conjointe enseignant-élèves, mésogenèse, topogenèse, chronogenèse

\section{Heterogenität und differentielle Erwartungen: Eine Annäherung mittels einer vergleichenden Didaktik}

\section{Zusammenfassung}

In diesem Beitrag wird der Begriff «Heterogenität» in einer besonderen Weise verwendet: Die Autorin und der Autor befassen sich in einer didaktischen Perspektive mit Schülerinnen und Schülern, von denen gesagt wird, dass sie «in Schwierigkeiten stecken». Ihr Ziel ist zu untersuchen, wie in einer Gruppe von 3. und 4. (HarmoS-)Klassen Lehrpersonen und Schülerinnen und Schüler während dem Mathematik-Unterricht (Zahlen und Subtrahieren) handeln, sei es im Dialog oder in ihren Interaktionen. Das Konzept des differenzierenden didaktischen Vertrags ermöglicht eine Analyse der Führungsart der Lehrpersonen sowie der verschiedenen Interaktionsarten. Speziell untersucht werden die 
Gliederung des Unterrichts mit der ganzen Klasse, der Zusammenhang zwischen dieser Gliederung und der Betreuung von Einzelschülerinnen und -schülern im Rahmen dieses Unterrichts sowie die Folgen für das tatsächliche Lernen der betroffenen Schülerinnen und Schüler.

Schlagworte: Differenzierender didaktischer Vertrag, Lehrer-Schüler-Interaktion, Mesogenese, Topogenese, Chronogenese

\section{Eterogeneità e differenziazione delle aspettative: un approccio di didattica comparativa}

\section{Riassunto}

Questo contributo si propone di rivisitare il concetto di eterogeneità in prospettiva didattica e in relazione ai fenomeni che riguardano gli allievi dichiarati "in difficoltà". L'obiettivo è esaminare, all'interno di classi di terza e quarta (HarmoS), l'interazione di docenti e allievi su compiti specifici (numero e sottrazione). Il concetto di contratto didattico differenziato permette di analizzare il funzionamento delle interazioni e la gestione dei docenti. In particolare viene messa a fuoco l'articolazione tra il trattamento individuale delle differenze tra allievi e il trattamento collettivo da parte del docente, nonché gli effetti sull'apprendimento degli allievi di questa gestione.

Parole chiave: Contratto didattico differenziato, azione congiunta docente-allievo, mesogenesi, topogenesi, cronogenesi

\section{Heterogeneity and differential expectations: a comparative didactic approach}

\section{Abstract}

This contribution suggests revisiting the concept of school heterogeneity from a didactical perspective by focusing on the phenomena concerning pupils considered to have "difficulties" and to be at risk. The purpose is to examine how, in regular classes of 3rd and 4th elementary school (Swiss HarmoS system), teacher and pupils interact about specific tasks (number and subtractions). The concept of differentiated didactic contract enables to analyze the interactions' functioning and the heterogeneity management. The contribution focuses on the links between individual and collective ways of managing students' differences, and shows differential effects on their learning.

Keywords: Differential didactic contract, teacher-pupils joint action, mesogenesis, topogenesis, chronogenesis 


\section{Annexe 1: Tableau synoptique de la séance de 3P (événements collectifs et observation de 2 élèves)}

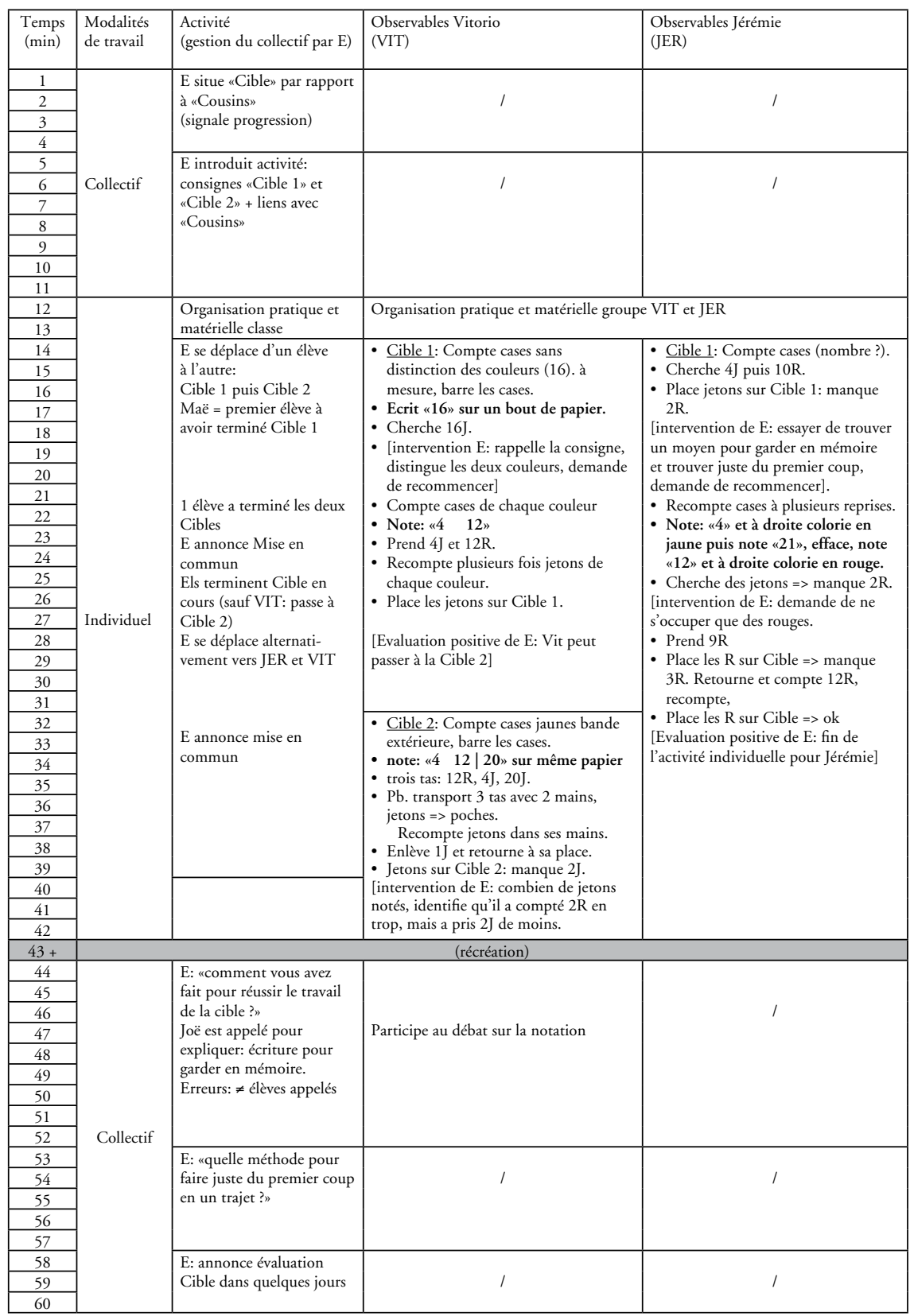




\section{Annexe 2: Tableau synoptique de la séance de 4P (événements collectifs et observation de 3 élèves)}

(les ${ }^{*}$ ou ${ }^{\circ} \mathrm{ou}+$ correspondent aux extraits qui suivent)

\begin{tabular}{|c|c|c|c|c|c|}
\hline \begin{tabular}{|l}
$\begin{array}{l}\text { Temps } \\
(\mathrm{min})\end{array}$ \\
\end{tabular} & \begin{tabular}{|l|} 
Modalités \\
de travail
\end{tabular} & Activité & $\begin{array}{l}\text { Observables } \\
\text { MAE } \\
\end{array}$ & $\begin{array}{l}\text { Observables } \\
\text { TOM } \\
\end{array}$ & $\begin{array}{l}\text { Observables } \\
\text { LAR }\end{array}$ \\
\hline 1 & \multirow[t]{3}{*}{ Collectif } & \multirow{3}{*}{$\begin{array}{l}\text { Lecture énoncé } \\
\text { problème } 1\end{array}$} & \multirow{3}{*}{ (V) } & \multirow{3}{*}{1001} & \multirow{3}{*}{ 步 } \\
\hline 2 & & & & & \\
\hline 3 & & & & & \\
\hline 4 & \multirow[t]{4}{*}{ Individuel } & \multirow{4}{*}{$\begin{array}{l}\text { Résolution du problème } \\
1 \text { sur table }\end{array}$} & \multirow{4}{*}{$\begin{array}{l}\text { is } \\
\text { it }\end{array}$} & \multirow{4}{*}{$38+90 \div 4=84$} & \multirow{4}{*}{ 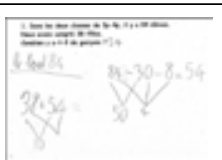 } \\
\hline 5 & & & & & \\
\hline 6 & & & & & \\
\hline 7 & & & & & \\
\hline 8 & \multirow[t]{13}{*}{ Collectif } & \multirow{3}{*}{$\begin{array}{l}\text { Façon de poser le } \\
\text { problème (pas la } \\
\text { solution) }\end{array}$} & \multirow{3}{*}{$\begin{array}{r}38 \\
+\ldots \ldots \\
84\end{array}$} & \multirow{3}{*}{ l } & \multirow{3}{*}{ l } \\
\hline 9 & & & & & \\
\hline 10 & & & & & \\
\hline 11 & & \multirow{4}{*}{$\begin{array}{l}\text { Examen de la solution du } \\
\text { Schtroumpf bricoleur: } \\
38 \\
+50 \\
\end{array}$} & \multirow{4}{*}{$\begin{array}{l}\text { Lève la main mais pas } \\
\text { interrogée par Ens }\end{array}$} & \multirow{4}{*}{\begin{tabular}{l}
\multicolumn{1}{c}{++} \\
Echange qui montre que \\
TOM a su donner la \\
différence entre 84 et 88 \\
à enlever, mais pas sur le \\
nbre de garçons
\end{tabular}} & \multirow{4}{*}{ l } \\
\hline 12 & & & & & \\
\hline 13 & & & & & \\
\hline 14 & & & & & \\
\hline 15 & & \multirow{6}{*}{$\begin{array}{l}\text { Examen de la solution du } \\
\text { Schtroumpf cuisinier: } \\
\\
\quad 38 \\
+46 \\
\end{array}$} & \multirow{6}{*}{$\begin{array}{l}\text { Echange qui montre que } \\
\text { MAE a su faire l'addition } \\
\text { avec retenue }\end{array}$} & \multirow{6}{*}{\begin{tabular}{|l|}
\multicolumn{1}{|c|}{} \\
\multicolumn{2}{|c|}{ Echange qui montre un } \\
retour sur la solution du \\
schtroumpf bricoleur au \\
regard de la solution du \\
schtroumpf cuisinier et \\
du débat à ce sujet $=>4$ \\
garçons de moins et non \\
pas de plus
\end{tabular}} & \multirow{6}{*}{$\begin{array}{l}\text { Echange avec LAR qui } \\
\text { montre calcul erroné de } \\
\text { LAR, mais pose correcte }\end{array}$} \\
\hline 16 & & & & & \\
\hline 17 & & & & & \\
\hline 18 & & & & & \\
\hline 19 & & & & & \\
\hline 20 & & & & & \\
\hline 21 & \multirow[t]{2}{*}{ Collectif } & \multirow{2}{*}{$\begin{array}{l}\text { Lecture énoncé } \\
\text { problème } 2\end{array}$} & \multirow{2}{*}{$\begin{array}{l}\text { MAE lit l'énoncé du pb. } \\
\Rightarrow>\text { pose pb en ligne et en } \\
\text { colonne }\end{array}$} & \multirow{2}{*}{$\begin{array}{l}\text { TOM est sollicité pour } \\
\text { dire les infos à disposition }\end{array}$} & \\
\hline 22 & & & & & I \\
\hline 23 & Individuel & Résolution du problème & 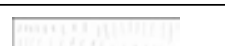 & 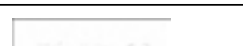 & 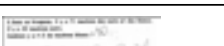 \\
\hline 24 & & & & $70 \cdot 31-2=39$ & \\
\hline 25 & & & 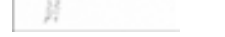 & & \\
\hline 26 & Collectif & Façon de poser le & & & оo \\
\hline 27 & & $\begin{array}{l}\text { probleme (pas la } \\
\text { solution) }\end{array}$ & 1 & 1 & $\begin{array}{l}\text { Echange avec LAR sur } \\
\text { pose du pb, tout, parties à }\end{array}$ \\
\hline 28 & & & & & trouver, noté au TN \\
\hline 29 & & $\begin{array}{l}\text { Solution Schtroumpf à } \\
\text { lunettes } 32\end{array}$ & & о0о & \\
\hline 30 & & & I & $\begin{array}{l}\text { Echange avec TOM sur } \\
\text { solution => erroné }\end{array}$ & I \\
\hline 31 & & Solution du & & 0000 & \\
\hline 32 & & Schtroumpt r & 1 & $\begin{array}{l}\text { Echange avec TOM sur la } \\
\text { base d'une comparaison des }\end{array}$ & (échange avec LAR sur \\
\hline 33 & & $\begin{array}{r}32 \\
+\quad 38\end{array}$ & & écritures & $\begin{array}{l}\text { calcul en ligne: } \\
71-30-2\end{array}$ \\
\hline 34 & & --.--- & & + échange avec Bar et Ens: & = calcul posé par LAR \\
\hline 35 & & & & $\begin{array}{l}\text { TOM souffle à Bar ce qu'il } \\
\text { s'agit de faire }\end{array}$ & $\begin{array}{l}\Rightarrow>\text { erreur de calcul, mais } \\
\text { correction collective }\end{array}$ \\
\hline 36 & & & & & \\
\hline 37 & & Clôture & l & 1 & l \\
\hline
\end{tabular}


